INPLASY

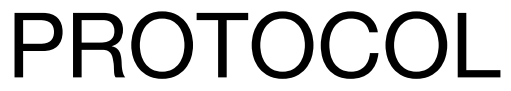

To cite: $X u$ et al. The protective effect of Baihui (DU 20)-based acupuncture for cerebral ischemia-reperfusion injury in rat models: a protocol for systematic review and metaanalysis. Inplasy protocol 202130114. doi: 10.37766/inplasy2021.3.0114

Received: 31 March 2021

Published: 31 March 2021

Corresponding author:

Fangyuan Xu

949599987@qq.com

Author Affiliation:

Hubei University of Chinese Medicine

Support: None.

Review Stage at time of this submission: Preliminary searches.

Conflicts of interest: None declared.

\section{The protective effect of Baihui (DU 20)-based acupuncture for cerebral ischemia-reperfusion injury in rat models: a protocol for systematic review and meta-analysis}

\author{
Xu, F1; Yang, Q2; Huang, W3; Liu, Z4; Lu, W5.
}

Review question / Objective: P: Rat models of cerebral ischemia-reperfusion which was established by middle cerebral artery occlusion (MCAO); I: Baihui-based acupuncture or electroacupuncture; C: Placebo, sham acupuncture, no treatment, traditional Chinese medicine or another active western medicine treatment; 0:The primary outcomes are shown as neurological function score and cerebral infarction size; S: randomized controlled trials.

Information sources: We will search the following electronic databases without restrictions for publication status: Chinese National Knowledge Infrastructure (CNKI), Wanfang database, Chinese Science and Technology Periodical Database (VIP), China Biology Medicine Database (CBM), PubMed, EMBASE, the Cochrane Library and Web of Science from inception to April 2021. In addition, we will also identify any additional qualified articles by searching reference lists of retrieved literatures.

INPLASY registration number: This protocol was registered with the International Platform of Registered Systematic Review and Meta-Analysis Protocols (INPLASY) on 31 March 2021 and was last updated on 12 April 2021 (registration number INPLASY202130114).

\section{INTRODUCTION}

Review question / Objective: P: Rat models of cerebral ischemia-reperfusion which was established by middle cerebral artery occlusion (MCAO); I: Baihui-based acupuncture or electroacupuncture; $C$ : Placebo, sham acupuncture, no treatment, traditional Chinese medicine or another active western medicine treatment; O:The primary outcomes are shown as neurological function score and cerebral infarction size; S: randomized controlled trials.

Condition being studied: Stroke is the second major cause of death and disability worldwide and the leading cause in China. 
A systematic review reported that there were $\mathbf{8 0 . 1}$ million cases of stroke occurring globally in 2016 and 13.7 million of which are new strokes. In particular, ischemic stroke accounts for $84.4 \%$ of the total number of strokes. As for the treatment of acute ischemic stroke, intravenous thrombolysis with recombinant tissue plasminogen activator and endovascular thrombectomy are the commonly used and effective surgical therapies. However, they can bring out complications such as hemorrhagic transformation and cerebral edema. Acupuncture, as a key component of traditional Chinese medicine (TCM), has become more and more popular in many countries. Moreover, acupuncture has shown the advantages of preventing and treating stroke in ancient times. So we will conduct this preclinical systematic review and meta-analysis to turn experimental studies into clinical benefits, which will do great help for the clinical promotion of acupuncture in the treatment of stroke.

\section{METHODS}

Participant or population: Rat models of cerebral ischemia-reperfusion which was established by middle cerebral artery occlusion (MCAO).

Intervention: Baihui-based acupuncture or electroacupuncture.

Comparator: Placebo, sham acupuncture, no treatment, traditional Chinese medicine or another active western medicine treatment.

Study designs to be included: Randomized controlled trials.

Eligibility criteria: P: Rat models of cerebral ischemia-reperfusion which was established by middle cerebral artery occlusion (MCAO) I: Baihui-based acupuncture or electroacupuncture $\mathrm{C}$ : Placebo, sham acupuncture, no treatment, traditional Chinese medicine or another active western medicine treatment. O:The primary outcomes are shown as neurological function score and cerebral infarction size.
Information sources: We will search the following electronic databases without restrictions for publication status: Chinese National Knowledge Infrastructure (CNKI), Wanfang database, Chinese Science and Technology Periodical Database (VIP), China Biology Medicine Database (CBM), PubMed, EMBASE, the Cochrane Library and Web of Science from inception to April 2021. In addition, we will also identify any additional qualified articles by searching reference lists of retrieved literatures.

Main outcome(s): Neurological function score and cerebral infarction size.

Quality assessment / Risk of bias analysis: Two reviewers will independently assess the methodological quality of the included literatures in accordance with the 10 items checklist of the Collaborative Approach to Meta-Analysis and Review of Animal Data from Experimental Studies : (1) peerreviewed publication; (2) statements of temperature control; (3) random allocation to experimental group or control group; (4) blinded induction of ischemia model; (5) blinded assessment of outcome; (6) use the anesthetic which presents no significant intrinsic neuroprotective activity; (7) appropriate animal model (aged, diabetic, or hypertensive); (8) sample size calculation; (9) compliance with animal welfare regulations; and (10) declaration of potential conflict of interests. The total quality score of each study was 10 points and the group median will be calculated.

Strategy of data synthesis: RevMan V.5.3.3 will be used for data analysis of the included studies. The $X^{2}$ test and I'statistic will be used to test the heterogeneity of the included literatures: when $\mathbf{P}<0.05$ or $\mathbf{I}^{2}>$ $50 \%$, it means that the heterogeneity between the studies is significant, and a random effects model will be used to synthesize the data. We should look for sources of heterogeneity actively. And the obvious clinical heterogeneity will be treated by the method of subgroup analysis or sensitivity analysis or just descriptive analysis. When $\mathrm{P}>0.05$ and $\mathrm{I}^{2}<50 \%$, use $\mathrm{a}$ fixed effects model. Dichotomous variables 
are expressed by relative risk (RR) with 95\% confidence intervals (Cls); continuous variables are expressed by mean difference(MD) or standardized mean difference (SMD) with $95 \%$ Cls.

Subgroup analysis: When sufficient data is available, we will conduct the following subgroup analysis to investigate the source of heterogeneity: ischemia time, reperfusion time, acupuncture methods and time, type of anesthetic drugs, treatment methods in the control group.

Sensitivity analysis: After conducting a quality assessment of the included studies, we can perform a sensitivity analysis if there exist studies of low quality. Sensitivity analysis will also be conducted when there exists considerable heterogeneity between the studies. We will remove the low-quality literature for further analysis to explore the source of heterogeneity, then we can acquire a stable result of our study.

Language: There is an English language summary and language limits will not be imposed on the search.

Country(ies) involved: China.

Keywords: acupuncture, Baihui acupoint, electroacupuncture, cerebral ischemiareperfusion injury, acute ischemic stroke, systematic review and meta-analysis.

Contributions of each author:

Author 1 - Fangyuan $\mathrm{Xu}$ drafted the manuscript.

Email: 949599987@qq.com

Author 2 - Qiqi Yang provided statistical expertise.

Email: 821920266@qq.com

Author 3 - Wei Huang read, provided feedback and approved the final manuscript.

Email: huangwei@hbhtcm.com

Author 4 - Zhenzhen Liu contributed to the development of the selection criteria, and the risk of bias assessment strategy.

Email: huangwei@hbhtcm.com

Author 5 - Wei Lu contributed to data curation.

Email: 921350785@qq.com 